\section{Dimethylformamid als Lösungsmittel bei der AAS-Analyse von Polymeren}

Dimethylformamide as a Solvent for AAS Analysis of Polymers

\section{OUIVIER}

Wissenschaftliches Laboratorium der Farbenfabriken Bayer AG, Dormagen

Eingegangen am 20. August 1971

Die Bestimmung von Metallspuren in Polymeren mit Hilfe der Atomabsorptions-Spektrophotometrie (AAS) bietet besonders dann Vorteile gegenüber anderen Verfahren, wenn die Polymeren in einem geeigneten Lösungsmittel gelöst und direkt analysiert werden können [1]. Bei der Verwendung von mit Wasser nicht mischbaren Lösungsmitteln taucht nun das Problem auf, daß man die üblicherweise in Wasser angesetzten Standards nicht zugeben kann. In einem Laboratorium mit einer breiten Palette zu analysierender Polymerer ist es technisch kaum möglich, die entsprechenden Standards in den verschiedensten Lösungsmitteln anzusetzen. Abgesehen vom Platzbedarf wächst anch die Fehlergefahr durch Verwechslungen.

Es konnte nun gezeigt werden, daß Dimethylformamid (DMF) nicht nur ein ausgezeichnetes Lösungsmittel für viele Polymere ist, sondern auch bei zahlreichen mit Wasser nicht mischbaren Systemen als Lösungsvermittler für die wäßrigen Standards dienen kann, ohne daß das gelöste Polymere wieder ausfällt. Schließlich sind Lösungsmittel, die an sich für die AAS schlecht geeignet sind (Aromaten, Halogenkohlenwasserstoffe), in Mischung mit DMF oft verwendbar.

Tab. 1 enthält eine Zusammenstellung der von uns untersuchten Polymeren, die in DMF löslich sind; in Tab. 2 sind Systeme angeführt, bei denen DMF als Lösungsvermittler dient. Die früher [1] bereits beschriebenen Systeme sind nur erwähnt, wenn Änderungen eingetreten sind.
Tabelle 1. In DMF lösliche Polymere

Celluloseacetat
Cellulosepropionat
Celluloseacetatbutyrat
Nitrocellulose
Polymethylmethacrylat
einige Polyurethane
Polyvinylalkohol (nur $1 \%$ ige Lösung)
Polyvinylchlorid (nur $1 \%$ ige Lösung)
Polyvinylehlorid/Polyvinylacetat-Copolymere

Tabelle 2. Systeme, bei denen DMF als Lösungsvermittler für die wäßrigen Standards wirkt

\begin{tabular}{ll}
\hline Polymeres & Lösungsmittel \\
\hline Polyäthylenterephthalat & Triäthylenglykol (Abbau) \\
Polystyrol & Methylisobutylketon \\
Polybutadien & Toluol \\
Polychloropren & Toluol \\
\hline
\end{tabular}

\section{Experimentelles}

Bezüglich Apparatur, Reagentien und Herstellung der Eichkurven gilt das früher [1] Beschriebene. Bei den in DMF löslichen Polymeren arbeitet man mit maximal 2\% igen Lösungen.

Für die in Tab. 2 angeführten Systeme hat sich folgende Arbeitsweise bewährt: Man stellt zunächst 10\% ige Polymerlösungen im angeführten Lösungsmittel her und verdünnt anschließend mit der 4 fachen Menge DMF. Polyäthylenterephthalat wird durch $30 \mathrm{~min}$ Erhitzen in siedendem Triäthylenglykol abgebaut. Die Bruchstücke sind im allgemeinen wasserlöslich. Es empfiehlt sich dennoch, mit DMF zu verdünnen, da die Versprühung in der Brennerkammer gleichmäßiger erfolgt.

Bei Polyvinylchlorid, Polystyrol, Polymethylmethacrylat und den von uns untersuchten Polyurethanen können nur $<1 \mathrm{ml}$ Wasser zugegeben werden, da sonst das Polymere ausfällt. Hier empfiehlt sich, die Standards durch Verdünnen der wäßrigen Stammlösung mit DMF anzusetzen.

\section{Literatur}

1. Olivier, M.: diese Z. 248, 145 (1969).

Dr. H. Olivier

Farbenfabriken Bayer A.G.

Werk Dormagen, Perlon-Abteilung

D-4047 Dormagen, Postfach 1140

Deutschland 